\title{
Shape of Crossover Between Mean-Field and Asymptotic Critical Behavior in a Three-Dimensional Ising Lattice
}

\author{
M. A. Anisimov ${ }^{1,2}$, E. Luijten ${ }^{3,4}$, V. A. Agayan ${ }^{1}$, J. V. Sengers ${ }^{1,2, *}$, and K. Binder ${ }^{4}$ \\ ${ }^{1}$ Institute for Physical Science and Technology, \\ University of Maryland, College Park, MD 20742, USA \\ ${ }^{2}$ Department of Chemical Engineering, University of Maryland, College Park, MD 20742, USA \\ ${ }^{3}$ Max-Planck-Institut für Polymerforschung, Postfach 3148, D-55021, Mainz, Germany \\ ${ }^{4}$ Institut für Physik, WA 331, Johannes Gutenberg-Universität, D-55099 Mainz, Germany
}

\begin{abstract}
Recent numerical studies of the susceptibility of the three-dimensional Ising model with various interaction ranges have been analyzed with a crossover model based on renormalization-group matching theory. It is shown that the model yields an accurate description of the crossover function for the susceptibility.
\end{abstract}

PACS: 05.70.Jk, 64.60.Fr

Keywords: Crossover critical phenomena; Ising model; Susceptibility

Recently, an accurate numerical study of the crossover from asymptotic (Ising-like) critical behavior to classical (mean-field) behavior has been performed both for two-dimensional [1,2] and three-dimensional [3] Ising systems in zero field on either side of the critical temperature with a variety of interaction ranges. It is the objective of the present work to analyze these numerical results within the framework of a crossover theory that is based on renormalizationgroup matching and that has already successfully been applied to the description of crossover in several experimental systems [4.5].

Qualitatively, the crossover is ruled by the parameter $t / G$ where $t=\left(T-T_{\mathrm{c}}\right) / T_{\mathrm{c}}$ is the reduced temperature distance to the critical temperature $T_{\mathrm{c}}$ and $G$ the Ginzburg number [6]. The Ginzburg number depends on the normalized interaction range $R$ as

$$
G=G_{0} R^{-2 d /(4-d)},
$$

where $d$ is the dimensionality of space and $G_{0}$ a constant. Hence, for $d=3$ the crossover occurs as a function of $t R^{6}$. Asymptotic critical behavior takes place for $t R^{6} \ll 1$ and classical behavior is expected for $t R^{6} \gg 1$. In real fluids the crossover is never completed in the critical domain (where $t \ll 1$ ), since the range of interaction is of the same order of magnitude as the distance between molecules $(R \simeq 1)$ 沺. A new Monte-Carlo algorithm, developed by Luijten and Blöte [7], offers the advantage that the ratio $t / G$ can be tuned over more than eight orders of magnitude allowing one to cover the full crossover region in three-dimensional spin models [3].

A sensitive description of crossover behavior is obtained from an analysis of the effective critical exponent of the susceptibility (the third derivative of the free energy), defined as

$$
\gamma_{\text {eff }}^{ \pm} \equiv-d \ln \hat{\chi} / d \ln |t|
$$

where the scaled susceptibility $\hat{\chi}=k_{B} T_{\mathrm{c}}(R)(\partial m / \partial h)_{T}, k_{\mathrm{B}}$ the Boltzmann constant, $m$ the order parameter, $h$ the ordering field, and where the "+" sign applies for $T>T_{\mathrm{c}}$, and the "-" sign for $T<T_{\mathrm{c}}$. As is seen from Figs. 1 and 2 , the variation of $\gamma_{\text {eff }}^{ \pm}$reproduces the Ising asymptotic critical behavior $\left(\gamma_{\text {eff }}^{ \pm} \simeq 1.24\right)$ at $t R^{6} \ll 1$ as well as the mean-field asymptote $\left(\gamma_{\mathrm{eff}}^{ \pm}=\gamma_{\mathrm{MF}}=1\right)$ at $t R^{6} \gg 1$. Apparently, all data would seem to collapse onto a universal function of the reduced variable $t R^{6}$ as predicted by a field-theoretical treatment [8,9] and by the $\varepsilon$-expansion [10]. However, as was noted in Ref. [3], a more careful look at the data reveals a remarkable discrepancy between the theoretical calculations [8 12] and the simulation results. Namely, the shape of the crossover is sharper than predicted by the theory [1, 12, especially for short ranges of interaction. We will show that this discrepancy is related to the findings of Refs. [4, 5], where it was shown that there is a fundamental problem in describing the crossover of $\gamma_{\text {eff }}^{ \pm}$by a universal function which contains only a single crossover parameter $G \propto R^{-6}$.

In zero-ordering field above $T_{\mathrm{c}}$ the susceptibility asymptotically close to the critical point behaves as

$$
\chi=\Gamma_{0} t^{-\gamma}\left(1+\Gamma_{1} t^{\Delta_{\mathrm{s}}}+\Gamma_{2} t^{2 \Delta_{\mathrm{s}}}+a_{1} t+\ldots\right),
$$

${ }^{*}$ Corresponding author. E-mail: sengers@ipst.umd.edu 
where $\gamma=1.239 \pm 0.002$ (see, e.g., Refs. 13,14] and references therein) and $\Delta_{\mathrm{s}}=0.504 \pm 0.008$ [15] are universal Ising critical exponents, and where $\Gamma_{0}, \Gamma_{1}, \Gamma_{2}$, and $a_{1}$ are system-dependent amplitudes. Expansion (3) is called the Wegner series [16].

In a universal single-parameter crossover theory [8, 10], the Ginzburg number is responsible both for the range of validity of the mean-field approximation and for the convergence of the Wegner series (3). However, it is known [17 19], that the sign of the first Wegner correction amplitude $\Gamma_{1}$ depends on the difference $u-u^{*}$, where $u$ is the scaled coupling constant and $u^{*}=0.472$ is the universal coupling constant at the Ising fixed point [20]. Moreover, Liu and Fisher [18] concluded that the three-dimensional nearest-neighbor Ising model has a negative leading Wegner correction amplitude $\Gamma_{1}$, so that $\gamma_{\text {eff }}^{ \pm}$asymptotically approaches $\gamma \simeq 1.24$ from above. Therefore, since the coupling constant itself depends on the interaction range, the shape of $\gamma_{\text {eff }}^{ \pm}$cannot be represented by a universal function of the Ginzburg number, since $G$ is not proportional to the difference $u-u^{*}$.

In this paper we therefore present an analysis of the numerical data for $\gamma_{\text {eff }}^{ \pm}$[3] in terms of a crossover model based on renormalization-group matching for the free-energy density [17,19,21]. This model contains two crossover parameters $\bar{u}=u / u^{*}$ and $\Lambda$ (a dimensionless cutoff wave number), and two rescaled amplitudes $c_{t}$ and $c_{\rho}$ related to the coefficients of the local density of the classical Landau-Ginzburg free energy $\Delta A$ :

$$
\begin{aligned}
\frac{v_{0}}{k_{\mathrm{B}} T} \frac{d(\Delta A)}{d V} & =\frac{1}{2} a_{0} \tau \varphi^{2}+\frac{1}{4 !} u_{0} \varphi^{4}+\frac{1}{2} c_{0}(\nabla \varphi)^{2} \\
& =\frac{1}{2} c_{t} \tau M^{2}+\frac{1}{4 !} u^{*} \bar{u} \Lambda M^{4}+\frac{1}{2}(\tilde{\nabla} M)^{2},
\end{aligned}
$$

with $\tau=\left(T-T_{\mathrm{c}}\right) / T, M=c_{\rho} \varphi=\left(a_{0} / c_{t}\right)^{1 / 2} \varphi, a_{0}=c_{\rho}^{2} c_{t}, u_{0}=u^{*} \bar{u} \Lambda c_{\rho}^{4}, c_{0}=c_{\rho}^{2} v_{0}^{2 / 3}$, and $\tilde{\nabla}=v_{0}^{1 / 3} \nabla$. The average molecular volume $v_{0}$ and the prefactor $v_{0} / k_{\mathrm{B}} T$ are introduced to make the free-energy density and all the coefficients dimensionless. The inverse crossover susceptibility $\chi^{-1}=\left(\partial^{2} \Delta \tilde{A} / \partial M^{2}\right)_{\tau}$, where $\Delta \tilde{A}$ is the crossover (renormalized) free-energy density, in zero field above $T_{\mathrm{c}}$ reads 田

$$
\chi^{-1}=c_{\rho}^{2} c_{t} \tau Y^{(\gamma-1) / \Delta_{\mathrm{s}}}(1+y)
$$

with

$$
y=\frac{u^{*} \nu}{2 \Delta_{\mathrm{s}}}\left\{2\left(\frac{\kappa}{\Lambda}\right)^{2}\left[1+\left(\frac{\Lambda}{\kappa}\right)^{2}\right]\left[\frac{\nu}{\Delta_{\mathrm{s}}}+\frac{(1-\bar{u}) Y}{1-(1-\bar{u}) Y}\right]-\frac{2 \nu-1}{\Delta_{\mathrm{s}}}\right\}^{-1},
$$

where $\nu \simeq 0.630$ 15]22] is the critical exponent of the asymptotic power law for the correlation length $\xi$ [4]. Note that $\chi^{-1}=\left(T_{\mathrm{c}} / T\right) \hat{\chi}^{-1}$ and the relation between $\gamma_{\mathrm{eff}} \equiv-d \ln \chi / d \ln |\tau|$ and $\gamma_{\text {eff }}^{ \pm}$, given by Eq. (2), is $\gamma_{\text {eff }}^{ \pm}=\gamma_{\text {eff }}+\left(1-\gamma_{\text {eff }}\right) \tau$, both above and below the critical temperature. The crossover function $Y$ is defined by

$$
1-(1-\bar{u}) Y=\bar{u}\left[1+\left(\frac{\Lambda}{\kappa}\right)^{2}\right]^{1 / 2} Y^{\nu / \Delta_{\mathrm{s}}}
$$

and is to be found numerically. The parameter $\kappa$ in Eq. (7) is inversely proportional to the fluctuation-induced portion of the correlation length and serves as a measure of the distance to the critical point. In zero field above $T_{\mathrm{c}}$ the expression for $\kappa^{2}$ reads:

$$
\kappa^{2}=c_{t} \frac{T}{T_{\mathrm{c}}} \tau Y^{(2 \nu-1) / \Delta_{\mathrm{s}}}=c_{t} t Y^{(2 \nu-1) / \Delta_{\mathrm{s}}} .
$$

We modified the original expression for $\kappa^{2}$, given by Eq. (3) in [ [ ], by introducing the non-asymptotic factor $T / T_{\mathrm{c}}$ in Eq. (8) so that $\kappa^{2}$ becomes infinite at $T \rightarrow \infty$ [23]. Asymptotically close to the critical point $(\Lambda / \kappa \gg 1)$, the following expression is obtained for the first correction amplitude $\Gamma_{1}$ in Eq. (3):

$$
\Gamma_{1}=g_{1}\left(\frac{\sqrt{c_{t}}}{\bar{u} \Lambda}\right)^{2 \Delta_{s}}(1-\bar{u}),
$$

where $g_{1} \simeq 0.62$ is a universal constant [21].

In the approximation of an infinite cutoff $\Lambda \rightarrow \infty$, which physically means neglecting the discrete structure of matter, $\bar{u}=u_{0} c_{t}^{2} /\left(u^{*} \Lambda a_{0}^{2}\right) \rightarrow 0$ and the two crossover parameters $\bar{u}$ and $\Lambda$ in the crossover equations collapse into a single one, $\bar{u} \Lambda$, which is related to the Ginzburg number $G$ by [21] 


$$
G=g_{0} \frac{(\bar{u} \Lambda)^{2}}{c_{t}}=g_{0} \frac{u_{0}^{2} v_{0}^{2}}{\left(u^{*}\right)^{2} a_{0}^{4} \bar{\xi}_{0}^{6}},
$$

where $g_{0} \simeq 0.028$ is a universal constant [21] and $\bar{\xi}_{0}=v_{0}^{1 / 3} c_{t}^{-1 / 2}=\left(c_{0} / a_{0}\right)^{1 / 2}$ is the mean-field amplitude of the power law for the correlation length. Note that the Ginzburg number does not depend explicitly on the cutoff $\Lambda$ or on $\bar{u}$. This single-parameter crossover, i.e., the crossover for $\bar{u}=0$, is universal and is indicated in Fig. 1 by a dashed-dotted curve. This simplified description of the crossover is equivalent to the results of Bagnuls and Bervillier [9] and of Belyakov and Kiselev 10].

In the simulations 31, each spin interacts equally with its $z$ neighbors lying within a distance $R_{\mathrm{m}}$ on a threedimensional cubic lattice. The effective range of interaction $R$ is then defined as $R^{2}=z^{-1} \sum_{j \neq i}\left|\mathbf{r}_{i}-\mathbf{r}_{j}\right|^{2}$ with $\left|\mathbf{r}_{i}-\mathbf{r}_{j}\right| \leq R_{\mathrm{m}}$ 迥. We have approximated the relation between $R$ and $R_{\mathrm{m}}$ by $R^{2}=\frac{3}{5} R_{\mathrm{m}}^{2}\left(1+\frac{2}{3} R_{\mathrm{m}}^{-2}\right)$, as indicated in the insert in Fig. 3. In order to compare the numerical results to the theoretical prediction Eq. (5), we need the range dependence of the parameters $c_{t}$ and $\bar{u}$. Indeed, the asymptotic $R$ dependence of $\bar{u}$ follows directly from simple scaling arguments 值, $\bar{u}=\bar{u}_{0} R^{-4}$, and $c_{t}$ varies as its square root, $c_{t}=c_{t 0} R^{-2}$. For a three-dimensional simple cubic lattice, $\Lambda=\pi$ [18,24], and we obtain for the Ginzburg number

$$
G=G_{0} R^{-6}=0.28\left(\bar{u}_{0}^{2} / c_{t 0}^{4}\right) c_{t}^{3}=0.28\left(\bar{u}_{0}^{2} / c_{t 0}\right) R^{-6} .
$$

The nonuniversal parameters $c_{t 0}$ and $\bar{u}_{0}$ have to be determined from a least-squares fit to the numerical data for $\gamma_{\text {eff }}^{+}$, which yielded $c_{t 0}=1.72$ and $\bar{u}_{0}=1.22$ and hence $G_{0} \approx 0.24$. The solid lines in Fig. 1 indicate the corresponding theoretical curves. It should be noted that these curves are calculated for each value of $R_{m}$ separately; the piecewise continuous character of this description directly reflects the fact that the crossover cannot be described by a universal single-parameter function. Indeed, Fig. 1 also shows two attempts to describe the data in terms of such a function. The dash-dotted line corresponds to the limit $\bar{u} \rightarrow 0$, whereas the dotted curve corresponds to $\bar{u}_{0}=1.22$ and $\Lambda=\pi$ (a continuation of the theoretical curve for $R=1$ ). We see that the actual crossover lies between these two bounding curves, with $\bar{u} \simeq 0$ for large $R$ and $\bar{u} \simeq 1.2$ for $R=1$. Thus, it is clearly seen that without including the $R$ dependence of $\bar{u}$ it is impossible to describe data for short interaction ranges $R_{\mathrm{m}}^{2} \leq 5$. The dependence of $\bar{u}$ on $R$ is shown in Fig. 3. The two adjustable parameters $c_{t 0}$ and $\bar{u}_{0}$ are strongly correlated and if one of them is fixed at a predicted value, the quality of the description remains the same. We hence tried to fit the data while keeping $c_{t 0}$ fixed at the theoretically predicted value $\left.c_{t 0}=2 d=6 \sqrt{25}, 26\right]$. In this case a fit of the same quality is obtained with $\bar{u}_{0}=1.22$, provided that $\Lambda \simeq 2 \pi$. The value of $G_{0} \approx 0.24$ then remains unchanged.

To describe the data below the critical temperature, a connection between $M$ and $\tau$ in zero field is to be found from the condition $(\partial \Delta \tilde{A} / \partial M)_{\tau}=0$. The relation between $M$ and $\tau$ appears to be implicit and $\chi$ as a function of $\tau$ cannot be expressed in an explicit form either. Of course, the parameters $c_{t 0}$ and $\bar{u}_{0}$ should be the same as for $T>T_{\mathrm{c}}$ and we hence kept them fixed at the above-mentioned values. However, the parameter $G_{0}$ appearing in Eq. (11) will take a different value. We took this into account by introducing a factor $G_{0}^{+} / G_{0}^{-}$into the temperature scale: $t \rightarrow t \cdot\left(G_{0}^{+} / G_{0}^{-}\right)$. Figure 2 shows the results for $T<T_{\mathrm{c}}$, where the factor $G_{0}^{+} / G_{0}^{-}$was included as an adjustable parameter. Our estimate $G_{0}^{-} / G_{0}^{+}=2.58$ must be compared with the theoretical result $G_{0}^{-} / G_{0}^{+}=3.125$ 27]. Interestingly, $\gamma_{\text {eff }}^{-}$clearly shows a minimum around $|t| R^{6} \sim 10^{2}$. This corroborates the nonmonotonic character of the crossover of $\gamma_{\text {eff }}^{-}$, earlier observed for the two-dimensional Ising lattice [2], where the effect is much more pronounced. We note that already in Ref. [28] a field-theoretic calculation of the crossover in the low-temperature regime has been given (in the limit $\bar{u} \rightarrow 0$ ), but only recently this has been extended to cover the full crossover region [29]. Actually, also here a nonmonotonicity in $\gamma_{\text {eff }}^{-}$has been observed.

In summary, we remark that although in general the theory contains two crossover parameters $\bar{u}$ and $\Lambda$, only one parameter $(\bar{u})$ changes with the range of interaction. However, this does not mean that the crossover is a universal function of $t R^{6}$. Indeed, the effective range of interaction $R$ affects the behavior of $\gamma_{\text {eff }}^{ \pm}$in a twofold way: through the Ginzburg number, which is proportional to $c_{t}^{3}$, and through the first Wegner correction, with an amplitude $\Gamma_{1}$ that is proportional to $(1-\bar{u})$ [Eq. (9)]. Hence, there is no way to describe the data for short interaction ranges without allowing for $\bar{u}$ to become larger than unity and correspondingly $\Gamma_{1}$ to change its sign between $R_{\mathrm{m}}=2$ and $R_{\mathrm{m}}=1$ as indicated in Fig. 3. In previous publications we have shown that Eq. (5), derived from renormalization-group matching, gives an excellent representation of the experimentally observed crossover behavior in simple and complex fluids [4, [- [30]. From the evidence presented in this paper, we conclude that the same crossover model also yields a quantitative description of the crossover critical behavior of a three-dimensional Ising lattice.

\section{Acknowledgments}

We acknowledge valuable discussions with M. E. Fisher and assistance from A. A. Povodyrev. The research at the University of Maryland was supported by DOE Grant No. DE-FG02-95ER-14509. E. Luijten acknowledges the HLRZ Jülich for computing resources on a Cray-T3E. 
[1] E. Luijten, H. W. J. Blöte, and K. Binder, Phys. Rev. E 54 (1996) 4626.

[2] E. Luijten, H. W. J. Blöte, and K. Binder, Phys. Rev. Lett. 79 (1997) 561.

[3] E. Luijten and K. Binder, Phys. Rev. E 58 (1998) R4060.

[4] M. A. Anisimov, A. A. Povodyrev, V. D. Kulikov, and J. V. Sengers, Phys. Rev. Lett. 75 (1995) 3146.

[5] Y. B. Melnichenko, M. A. Anisimov, A. A. Povodyrev, G. D. Wignall, J. V. Sengers, and W. A. Van Hook, Phys. Rev. Lett. 79 (1997) 5266.

[6] A. Z. Patashinskii and V. L. Pokrovskii, Fluctuation Theory of Phase Transitions (Pergamon, Oxford, 1979).

[7] E. Luijten and H. W. J. Blöte, Int. J. Mod. Phys. C 6 (1995) 359.

[8] C. Bagnuls and C. Bervillier, J. Phys. Lett. (Paris) 45 (1984) L-95.

[9] C. Bagnuls and C. Bervillier, Phys. Rev. B 32 (1985) 7209.

[10] M. Y. Belyakov and S. B. Kiselev, Physica A 190 (1992) 75.

[11] P. Seglar and M. E. Fisher, J. Phys. C 13 (1980) 6613.

[12] M. E. Fisher, Phys. Rev. Lett. 57 (1986) 1911.

[13] J. V. Sengers and J. M. H. Levelt Sengers, Ann. Rev. Phys. Chem. 37 (1986) 189.

[14] A. J. Liu and M. E. Fisher, Physica A 156 (1989) 35.

[15] R. Guida and J. Zinn-Justin, J. Phys. A 31 (1998) 8103.

[16] F. J. Wegner, Phys. Rev. B 5 (1972) 4529.

[17] J.F. Nicoll and P. C. Albright, Phys. Rev. B 31 (1985) 4576.

[18] A. J. Liu and M. E. Fisher, J. Stat. Phys. 58 (1990) 431.

[19] S. Tang, J. V. Sengers, and Z. Y. Chen, Physica A 179 (1991) 344.

[20] J. C. Le Guillou and J. Zinn-Justin, J. Phys. (Paris) 48 (1987) 19.

[21] M. A. Anisimov, S. B. Kiselev, J. V. Sengers, and S. Tang, Physica A 188 (1992) 487.

[22] H. W. J. Blöte, E. Luijten, and J. R. Heringa, J. Phys. A 28 (1995) 6289.

[23] A. Kostrowicka Wyczalkowska, M. A. Anisimov, and J. V. Sengers, Fluid Phase Equilibria $158-160$ (1999) 523.

[24] M. E. Fisher, Rev. Mod. Phys. 46 (1974) 597.

[25] M. E. Fisher and R. J. Burford, Phys. Rev. 156 (1967) 583.

[26] E. Luijten, unpublished results.

[27] M. Lifschitz, J. Dudowicz, and K. F. Freed, J. Chem. Phys. 100 (1994) 3957.

[28] C. Bagnuls, C. Bervillier, D. .I. Meiron, and B. G. Nickel, Phys. Rev. B 35 (1987) 3585.

[29] A. Pelissetto, P. Rossi, and E. Vicari, Phys. Rev. E 58 (1998) 7146.

[30] J. Jacob, A. Kumar, M. A. Anisimov, A. A. Povodyrev, and J. V. Sengers, Phys. Rev. E 58 (1998) 2188. 


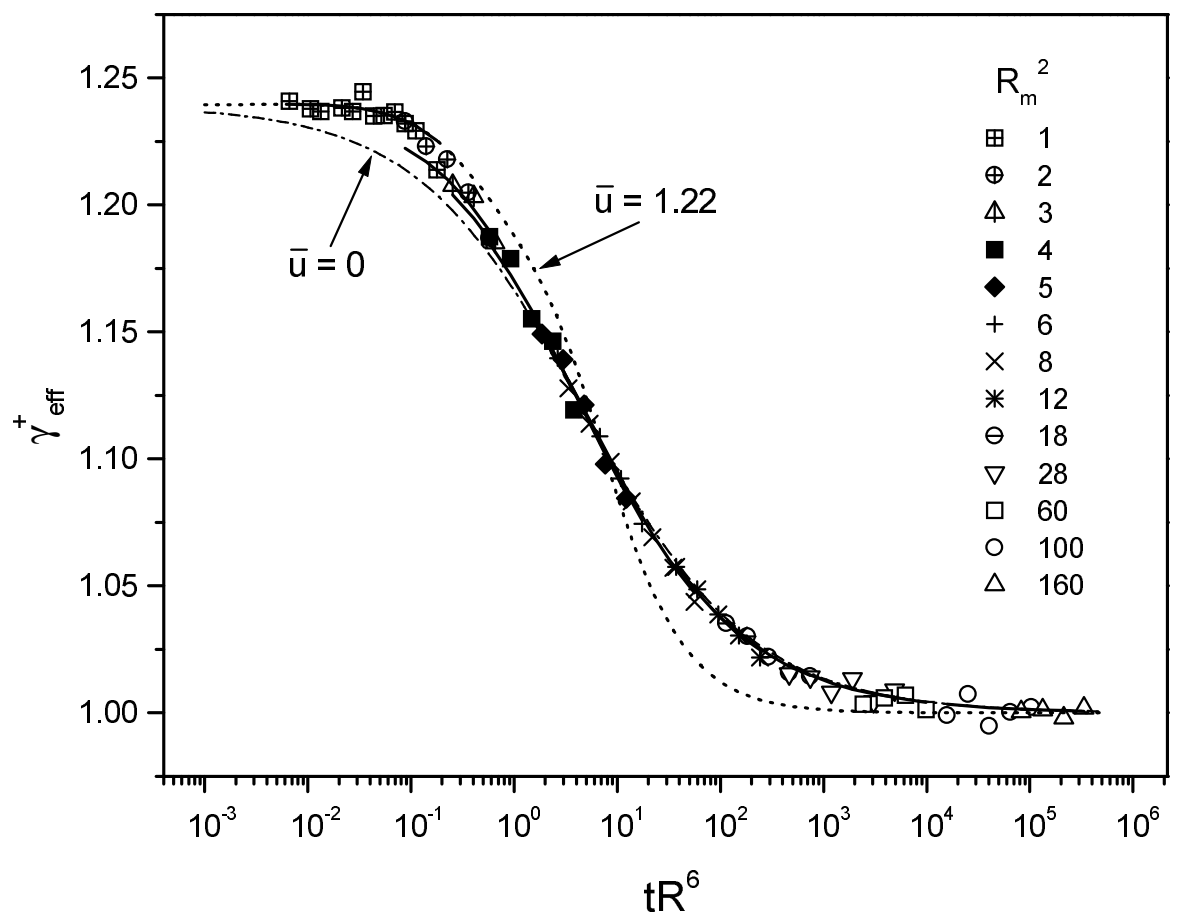

FIG. 1. The effective susceptibility exponent $\gamma_{\text {eff }}^{+}$above $T_{\mathrm{c}}$. The symbols indicate numerical simulation data [3]. The solid curves represent values calculated from Eq. (5). The dashed-dotted curve corresponds to the limit $\bar{u} \rightarrow 0$. The dotted curve is a continuation of the crossover curve for $\bar{u}=1.22$. For clarity, the error bars have been omitted; they are all of the order of 0.004 .

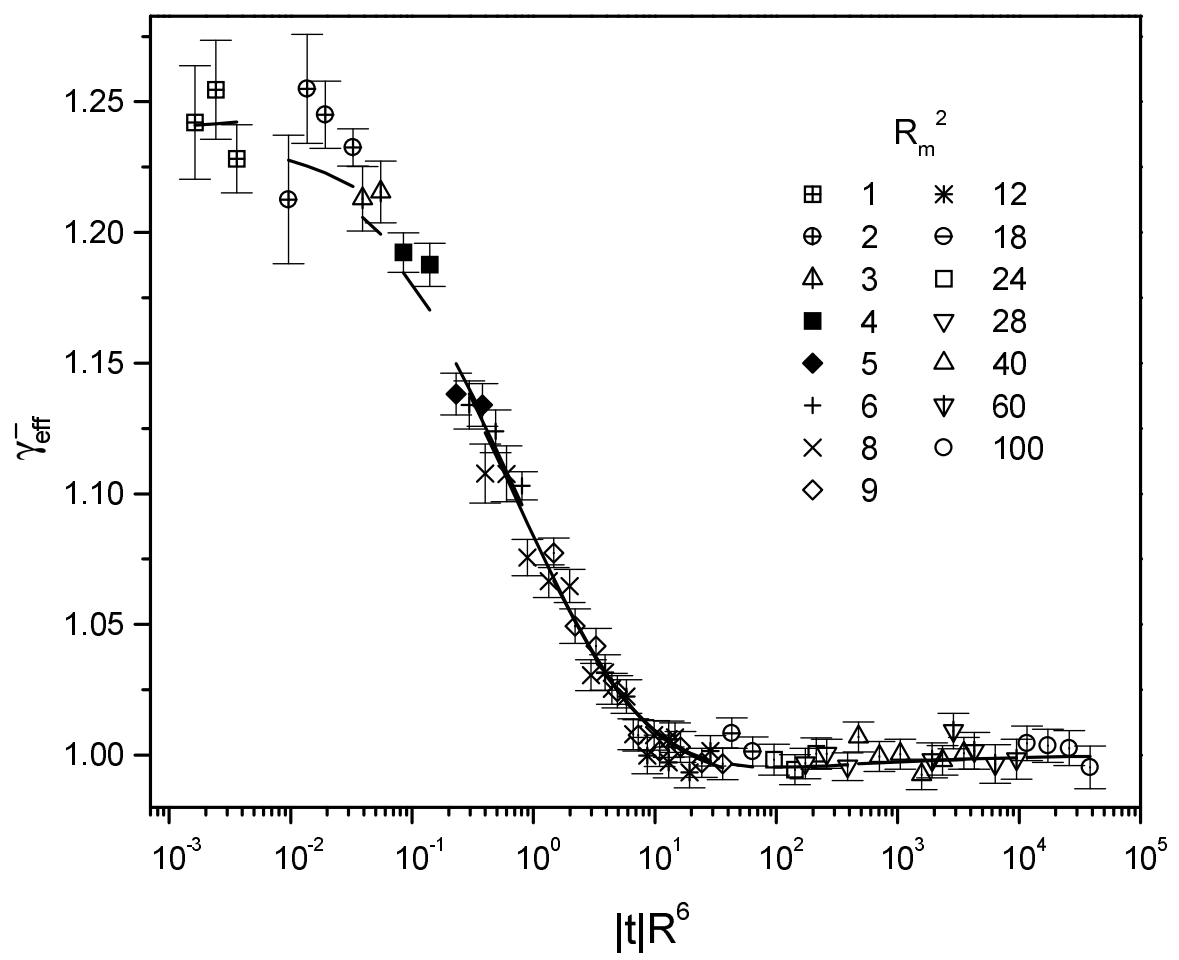

FIG. 2. The effective susceptibility exponent $\gamma_{\text {eff }}^{-}$below $T_{\mathrm{c}}$. The symbols indicate numerical simulation data [3]. The solid curves represent values calculated from the renormalization-group matching crossover model. 


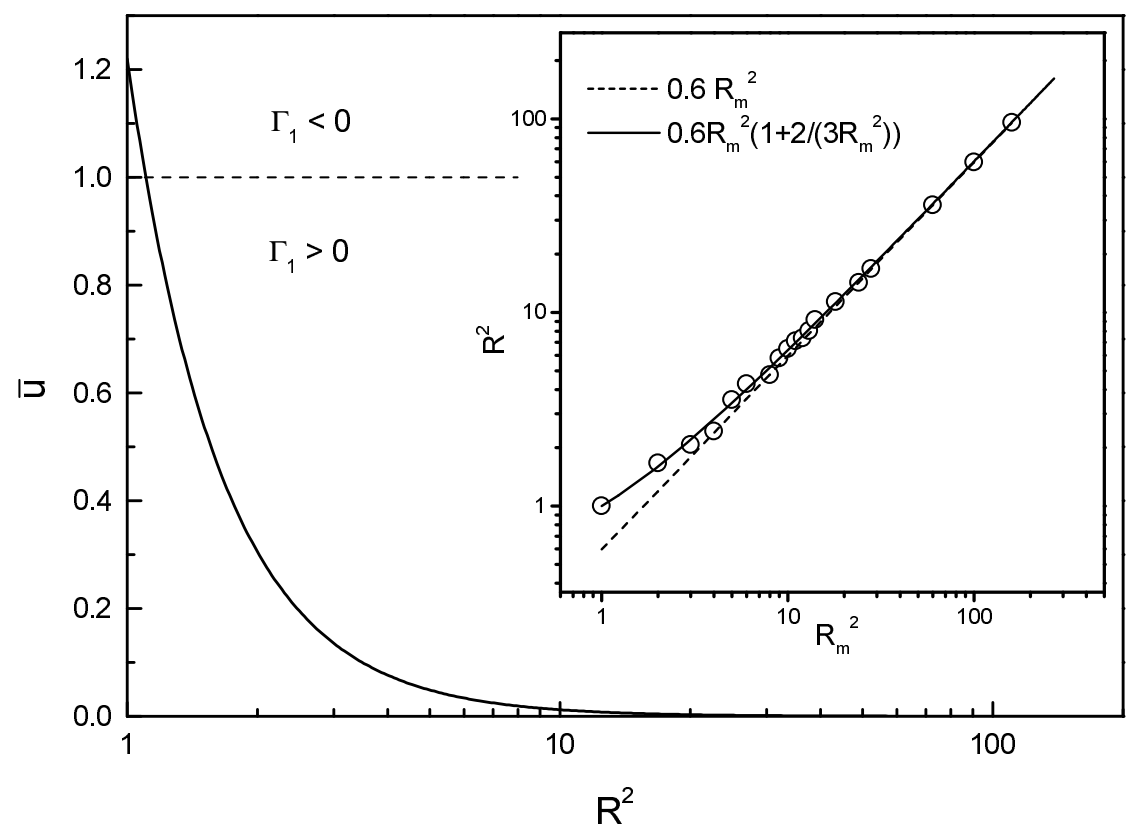

FIG. 3. Dependence of the normalized coupling constant $\bar{u}$ on the normalized interaction range $R$. Note that $\bar{u}$ becomes larger than unity for very short interaction ranges. Insert: Effective range of interaction $R$ (open circles) plotted as a function of $R_{\mathrm{m}}$. The solid line corresponds to the approximation mentioned in the text and the dashed line represents the asymptotic behavior for large $R$. 